\title{
Argemone mexicana decoction versus artesunate-amodiaquine for the management of malaria in Mali: policy and public-health implications
}

\author{
Bertrand Graz ${ }^{\mathrm{a}, \mathrm{b}, *}$, Merlin L. Willcox ${ }^{\mathrm{a}, \mathrm{b}, \mathrm{f}}$, Chiaka Diakite ${ }^{\mathrm{b}, \mathrm{c}}$, Jacques Falquet ${ }^{\mathrm{a}, \mathrm{b}}$, \\ Florent Dackuo ${ }^{\mathrm{d}}$, Oumar Sidibe ${ }^{\mathrm{b}, \mathrm{c}}$, Sergio Giani $^{\mathrm{e}}$, Drissa Diallo ${ }^{\mathrm{c}}$ \\ a Antenna Technologies, Geneva, Switzerland \\ b The Research Initiative on Traditional Antimalarial Methods, 66 Lye Valley, Oxford OX3 7ER, UK \\ c Departement de Medecine Traditionnelle, Bamako, Mali \\ d Faculte de Medecine, Pharmacie et Odonto-Stomatologie, Universite de Bamako, Bamako, Mali \\ e Aidemet, Bamako, Mali \\ ${ }^{\mathrm{f}}$ Department of Primary Health Care, University of Oxford, UK
}

\section{A R T I C L E I N F O}

\section{Article history:}

Received 19 December 2008

Received in revised form 13 July 2009

Accepted 14 July 2009

Available online 5 September 2009

\section{Keywords:}

Malaria

Herbal medicine

Argemone mexicana

Home-based management

Artemisinin combination therapy Mali

\begin{abstract}
A B S T R A C T
A classic way of delaying drug resistance is to use an alternative when possible. We tested the malaria treatment Argemone mexicana decoction (AM), a validated self-prepared traditional medicine made with one widely available plant and safe across wide dose variations. In an attempt to reflect the real situation in the home-based management of malaria in a remote Malian village, 301 patients with presumed uncomplicated malaria (median age 5 years) were randomly assigned to receive AM or artesunate-amodiaquine [artemisinin combination therapy (ACT)] as first-line treatment. Both treatments were well tolerated. Over 28 days, second-line treatment was not required for $89 \%(95 \% \mathrm{CI} 84.1-93.2)$ of patients on AM, versus 95\% (95\% CI 88.8-98.3) on ACT. Deterioration to severe malaria was $1.9 \%$ in both groups in children aged $\leq 5$ years (there were no cases in patients aged $>5$ years) and $0 \%$ had coma/convulsions. AM, now government-approved in Mali, could be tested as a firstline complement to standard modern drugs in high-transmission areas, in order to reduce the drug pressure for development of resistance to ACT, in the management of malaria. In view of the low rate of severe malaria and good tolerability, AM may also constitute a first-aid treatment when access to other antimalarials is delayed.
\end{abstract}

(c) 2009 Royal Society of Tropical Medicine and Hygiene. Published by Elsevier Ltd. All rights reserved.

\section{Introduction}

How could modern antimalarials such as artemisinin combination therapies (ACTs) be kept effective for as long as possible, when threats of resistance are appearing? Widespread resistance to amodiaquine and sulphadoxinepyrimethamine already exists. ${ }^{1,2}$ There is also some

\footnotetext{
* Corresponding author. Present address: IUMSP-UEPP, Bugnon 17-1000 Lausanne, Switzerland. Tel.: +41 2131469 46; fax: +41213147244.

E-mail address: bertrand.graz@chuv.ch (B. Graz).
}

evidence of resistance developing to lumefantrine in areas where artemether-lumefantrine is widely used. ${ }^{3}$ Resistance will continue to spread, particularly to the longacting partner drugs, if ACTs are squandered on patients who do not absolutely need them.

Because $80 \%$ of malaria patients never go to a health facility, and treat themselves at home, ${ }^{4}$ WHO has been promoting the home-based management of malaria (HMM) with ACTs as first-line treatment. ${ }^{5,6}$ In most of subSaharan Africa, diagnostic facilities are not available or cost effective, so cases of fever are treated presumptively, 7,8 although many of these patients may not need treatment with an ACT. If the HMM strategy is implemented, it will 


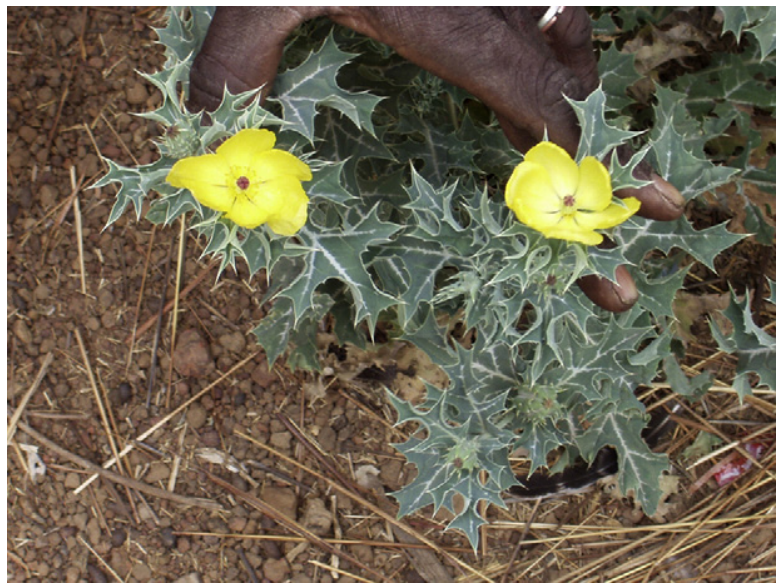

Figure 1. Argemone mexicana L. (Papaveraceae). Photo by $\mathrm{Dr}$ Jacques Falquet.

lead to the very widespread use of ACTs, and a greatly increased risk of resistance.

A classic way of delaying resistance is to use an alternative for all but the most vulnerable cases. Local, traditional resources might be an interesting solution if they have been in use for several generations and are still clinically effective locally, as it is probable that they will not lose their effectiveness rapidly. A strategy for a semi-immune population, using such a traditional product as first line with an ACT as second line, would reduce the likelihood of, and speed of, resistance developing to the ACT. In fact, up to $75 \%$ of malaria patients choose to treat themselves with herbal remedies. ${ }^{9}$

These observations and reflections led to the elaboration of the following research question: Could some local, traditional antimalarials be of interest for the management of malaria?

In an ethnomedical study in Mali, among the 66 plants used for malaria, eight were consistently associated with good clinical outcomes. ${ }^{10,11}$ These were then tested in vitro, and the most active happened to be the one with the best clinical correlates, a weed found in many tropical areas: Argemone mexicana L (Papaveraceae) (Figure 1).

Argemone mexicana is a pantropical weed with a long history of use in traditional medicine, dating back to the Aztecs. ${ }^{12}$ It is used as an antimalarial in several African countries, including Benin, Mali and Sudan. ${ }^{13-15}$ Its in-vitro efficacy against Plasmodium falciparum has been confirmed: the $\mathrm{IC}_{50}$ (concentration which inhibits $50 \%$ of parasites) values of the aerial parts of this plant, against the chloroquine-resistant $\mathrm{K} 1$ strain of $P$. falciparum, were 5.89 and $1.00 \mu \mathrm{g} / \mathrm{ml}$ for the aqueous decoction and methanol extracts, respectively. ${ }^{11}$ A prospective dose escalating clinical trial of Argemone mexicana decoction (AM) following a local recipe, in patients with uncomplicated malaria, found a dose-response correlation and a good safety profile. ${ }^{16}$ In addition to its promising profile, we observed that AM was often readily available where and when modern drugs of acceptable quality were not. ${ }^{11}$
The purpose of this study was to compare two strategies for the home-based management of presumed uncomplicated malaria: AM as first line with ACT as second line, versus ACT as first line.

\section{Materials and methods}

When designing this research project, the dilemma was whether to design a randomised controlled trial (RCT) in standard experimental conditions or to study the real situation (at least, 'as real as possible'). A standard RCT with patients meeting WHO research criteria would have provided an estimate of the magnitude of outcome differences between ACT and AM in an experimental setting. However, Malian medical and public health authorities were more interested in a real-situation estimate, the primary aim being to produce the basic knowledge necessary for issuing a new 'médicament traditionnel amélioré' (improved traditional drug), with a reasonable estimate of the effects to be anticipated when used outside of clinical settings and, most of the time, without laboratory diagnosis. So we had to agree a priori (based on health-policy grounds) what difference between AM and ACT would be acceptable in a 'real-life' setting (= delta). We then designed a study to test whether the observed difference was greater or smaller than the set delta.

\subsection{Setting}

This study was conducted in conditions as close to the 'real world' as possible, in the village of Missidougou, in the southeast of Mali. The village is located $40 \mathrm{~km}$ from the nearest Malian primary health centre, and $70 \mathrm{~km}$ from the nearest hospital (Sikasso). This includes a $25 \mathrm{~km}$ dirt track, along which there is no public transport. The village chief of Missidougou is a traditional healer, and his son has trained both with him and as a health-care assistant. He fulfils the role of a village health worker. The trial was undertaken during the peak malaria season, from late July to early December, 2006.

\subsection{Participants}

Patients first consulted the village health worker. He referred to the study team all patients whom he presumed to have malaria (mainly on the basis of a history of fever and no other obvious disease). The study was then explained to the patients. There was an information sheet, which was read and translated to the patients and their parents in the local language, as most were illiterate. Subjects (or their parents in the case of children) gave individual written consent, usually with a fingerprint, as most could not sign their name.

We included as many patients as possible with presumptive malaria, in order to obtain a realistic assessment of management in the hands of a village health worker. The exclusion criteria were: (1) severe malaria; (2) history of ingestion of a full dose of antimalarial on the same day; (3) lack of consent; (4) inability to return for follow-up; (5) ingestion of antimalarials not prescribed by the study team during the course of follow-up. 
Patients whom the village health worker or traditional healer could not treat were referred to the medical team for a consultation.

\subsection{Design}

Patients were randomly assigned to two groups, AM or ACT, using a computer-generated random number table and a 2:1 randomisation ratio with blocks of 6 stratified for age ( $<1$ year, $1-5$ years, $>5$ years $).{ }^{17}$ Owing to the very different nature of the two treatments, it was not possible to blind the patients or the clinicians to the treatments being taken. A small survey was undertaken to record treatment preferences in the research team and villagers.

Patients were followed up on days 3, 7, 14 and 28 and were advised to return at any time if they experienced a deterioration of their condition, or recurrence of symptoms. Patients were assessed by a medical doctor (history and physical examination). Thin and thick blood films were taken at baseline and at every follow-up visit. Haematocrit was measured at baseline and on day 28 , and in cases of treatment failure.

\subsection{Treatment interventions}

\subsubsection{AM group}

Argemone mexicana aerial parts were harvested and dried in the shade, mostly in the spring of 2006. The plant is very well known locally, and the risk of confusion with other species is very low, thanks to its characteristic yellow flowers, spiny leaves and yellow milky latex (Figure 1). Patients were given a bag of $500 \mathrm{~g}$ of the dried plant material, and the method of preparation was explained to them (or to the accompanying person, in the case of children) by the village health worker (who is also a traditional healer). Patients were advised to divide the dried herb into two parts, and to boil one part in about 2.51 of water (just enough to cover the dried material) for about $3 \mathrm{~h}$, then filter and keep in a clean, covered pot for a maximum of 4 days. The recommended regimen is a twice-daily dose, ranging in volume from a tea glass (ca. $60 \mathrm{ml}$ ) for infants to a coffee glass (ca. $300 \mathrm{ml}$ ) for adults. It is also recommended, for infants and small children, to apply the preparation to the skin ('washing') twice a day.

Patients were advised to take this treatment for 1 week. If at the end of the first week the patient had largely improved but was still experiencing a few symptoms such as a mild fever, they were given the treatment for a second week.

In cases of treatment failure, patients were given oral artesunate/amodiaquine, or intramuscular artemether if unable to swallow, or referred if appropriate. Treatment failure was defined as a worsening of the clinical condition, development of danger signs (inability to drink or breastfeed; vomiting everything; recent history of convulsions; lethargy or unconsciousness; prostration), or failure to improve with persistent fever.

\subsubsection{ACT group}

Patients were given artesunate $(50 \mathrm{mg})$ and amodiaquine $(153 \mathrm{mg})$ tablets in a co-blister pack (Arsucam,
Sanofi-Synthelabo Group, Casablanca, Morocco) by the village health worker. The dose was determined by the patient's weight, according to the following dosage schedule: $<10 \mathrm{~kg}, 1 / 2+1 / 2 ; 10-20 \mathrm{~kg}, 1+1 ; 21-40 \mathrm{~kg}, 2+2$; $>40 \mathrm{~kg}, 4+4$, twice daily for 3 days. For small children the tablets were crushed and mixed with sugar and a small amount of water. Women known to be pregnant at baseline were treated with sulphadoxine-pyrimethamine (three tablets in a single dose) instead of artesunateamodiaquine. In cases of treatment failure patients were given oral artemether-lumefantrine (Coartem Novartis, Basel, Switzerland), or intramuscular artemether if unable to swallow, or were referred if appropriate.

\subsubsection{Both groups}

The first administration of the medicine was observed in most cases, especially in patients who complained of vomiting. Sugar was added to increase palatability for young children. The dose was repeated if the patient vomited within $30 \mathrm{~min}$. If vomiting persisted the patient was given an intramuscular injection of artemether.

Any necessary additional treatment was given by the village health worker and the medical team as appropriate, and was recorded in the patient's notes. In cases of acute severe illness (whether or not related to malaria) patients were referred to the regional hospital.

\subsection{Laboratory methods}

A field laboratory was installed in the village, using solar panels and car batteries to power a microscope and centrifuge. Blood films were stained with $3 \%$ Giemsa for $40 \mathrm{~min}$, and parasite counts were done using standard $\mathrm{WHO}^{7}$ criteria. All blood films taken at baseline and on the day of a presumed new episode of malaria were checked by a second experienced microscopist (MLW). In addition, quality control was undertaken by a third independent expert microscopist (Dr Mouctar Diallo, Malaria Research and Training Centre, Bamako, Mali). Blood films at day 28 with negative or low parasitaemia $(<500 / \mathrm{mcl})$ were also double checked. As the slide may contain only one or two parasites, which may be spotted by only one microscopist, we decided to analyse two scenarios for parasitaemia at day 28: 'upper' estimate (taking the slide to be positive if even a single parasite was seen by one microscopist) and 'lower' estimate (taking the slide to be negative unless both microscopists confirmed the presence of malaria parasites).

Haematocrit was measured using microhaematocrit tubes in a centrifuge. PCR was not performed because of financial constraints.

Analyses of parasitaemia and haematocrit were also used to confirm whether or not the patient met the formal WHO definitions for severe malaria. ${ }^{7}$

\subsection{Outcome measures}

Results were analysed by intention to treat. Patients were excluded in the course of the study only if they withdrew consent or if they took a course of antimalarial medication that had not been prescribed by the study team. 
The primary endpoint for comparison was 'clinical recovery' at day 28 without need for re-treatment with the second-line antimalarial. Secondary endpoints were: absence of fever at days 14 and 28, incidence of severe malaria (which is a predictor of malaria mortality), ${ }^{18,19}$ incidence of new episodes of malaria, change in haematocrit between days 0 and 28 , incidence and severity of adverse events, and parasitaemia at day 28 .

\subsection{Statistical analysis}

The study was designed as a 'non-inferiority' trial. The non-inferiority range between the two strategies (= 'minimally important difference', in terms of health policy) was set after consultation with national health authorities (malaria and public-health programmes) at $15 \%$ for the primary endpoint.

Sample size was computed in order to assess with $80 \%$ power and 95\% confidence level that AM is 'non-inferior' to ACT, i.e. according to the set non-inferiority range of $15 \%$ and incidence assumptions of at least $85 \%$ not needing re-treatment with $\mathrm{ACT}$. Data were entered and analysed using Epi Info 5.01 (CDC, Atlanta, GA, USA) and SPSS 11.0 (SPSS Inc., Chicago, IL, USA) software. Comparison of groupspecific results were assessed using the $\chi^{2}$ test for linear trend. Multivariate analysis of potential confounding factors (mainly age, parasitaemia, haematocrit and baseline temperature) was not performed because there was no significant imbalance between the groups at baseline.

\subsection{Ethical issues}

The toxicological assessment of AM was completed according to international recommendations ${ }^{20}$ and national regulations. Patients were included only if they (or in the case of children, their parents) gave written informed consent. They were free to withdraw consent at any time. Patients were followed closely, and were given alternative treatment if necessary. Patients who did not return for follow-up were visited at home. All treatments were provided free of charge, including referral to hospital when necessary.

\section{Results}

\subsection{Patients}

From 29 July to 8 September 2006, 301 patients were included in the study. Twelve patients were excluded at baseline: six because of severe malaria; three were unable to return for follow-up; two refused to give consent; and one had started a course of antimalarial treatment the same day.

Of the 301 patients included, 199 were randomly assigned to the AM group and 102 to the ACT group (ratio 2:1). Three were excluded before day 14 (two took another antimalarial, one withdrew consent) and one on day 24 (took another antimalarial). For the purposes of the analyses below, the first three patients have been excluded from all analyses, and the fourth only from analyses of variables at day 28. No patients were lost to follow-up (Figure 2).

\subsection{Baseline characteristics}

As shown in Table 1, there were no important differences between the two groups in baseline characteristics. Many children had no measured fever at the time of inclusion, despite a diagnosis of malaria by the village health worker.

The blood film was positive for malaria parasites in the majority of patients (87-88\%). Of those with a positive blood film (257), only six (2\%) did not have a pure $P$. falciparum infection. Four had a mixed infection ( $P$. falciparum + another species), one had a $P$. malariae infection

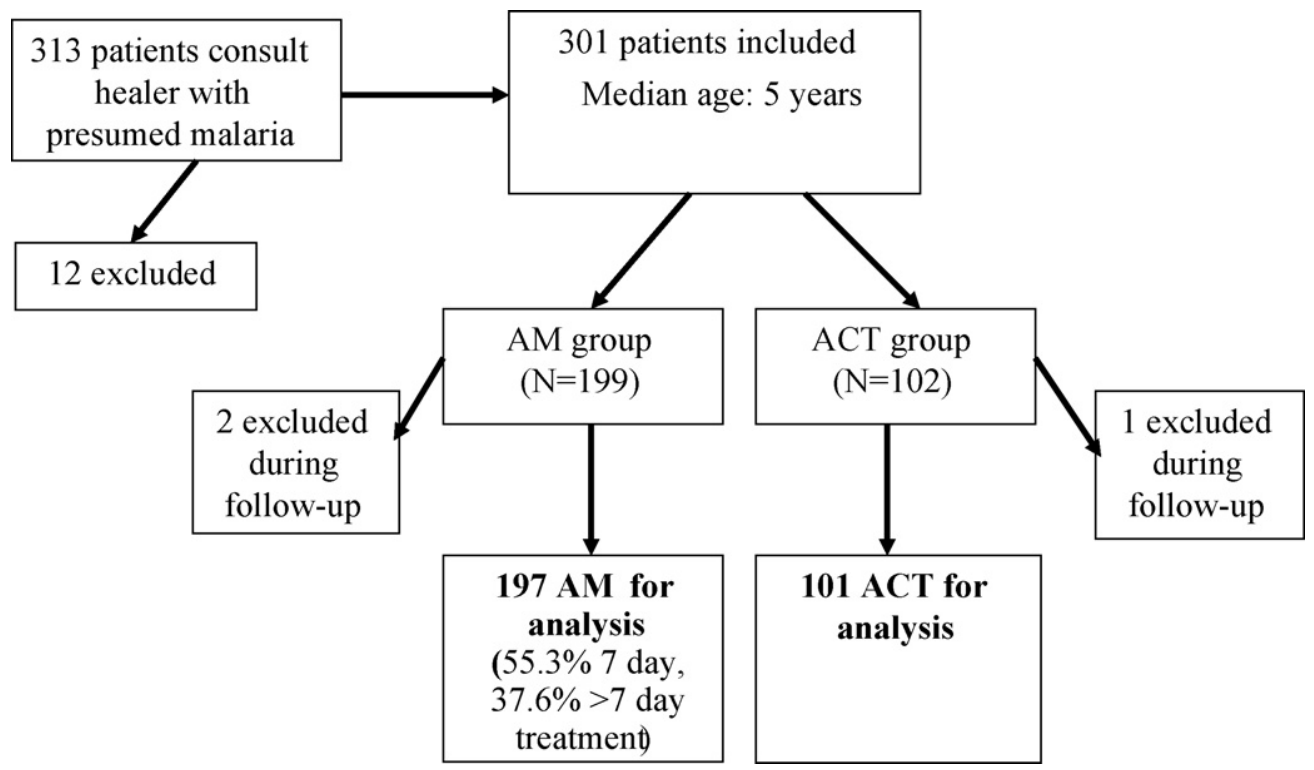

Figure 2. Flow of patients (with a 2:1 randomisation ratio). 
Table 1

Baseline characteristics of 298 patients, all diagnosed as 'malaria patients' by the village health worker

\begin{tabular}{|c|c|c|c|}
\hline & AM group $(\mathrm{n}=197)^{\mathrm{a}}$ & ACT group $(\mathrm{n}=101)^{\mathrm{a}}$ & $P$-value \\
\hline Sex (\% of males) & 49.2 & 42.6 & 0.3 \\
\hline Median age (years) (quartiles) & $5(2-20)$ & $5(2-20)$ & 0.6 \\
\hline Median weight (kg) (quartiles) & $18.0(9-55)$ & $18.0(10-46)$ & 0.9 \\
\hline Median weight/age ratio (kg/years) & 5.45 & 5.45 & 0.3 \\
\hline $\begin{array}{l}\text { Patients having taken modern antimalarial } \\
\text { treatment }(\%)\end{array}$ & 7.1 & 8.9 & 0.7 \\
\hline Splenomegaly present (\%) & 15.2 & 9.9 & 0.2 \\
\hline Median temperature $\left({ }^{\circ} \mathrm{C}\right)$ (quartiles) & $36.7(36.2-37.6)$ & $37.0(36.4-37.6)$ & 0.3 \\
\hline Temperature $<37.5^{\mathrm{b}}$ at day 0 & $71.9 \%$ & $64.7 \%$ & 0.2 \\
\hline Thick film positive (\%) & 87.8 & 87.1 & 0.7 \\
\hline $\begin{array}{l}\text { Geometric mean parasite count in patients with } \\
\text { positive thick film (per } \mathrm{mcl} \text { ) (interquartile } \\
\text { range) }\end{array}$ & $893(85-10641)$ & $1014(76-11614)$ & 0.8 \\
\hline Haematocrit (\%) & $34.3[155]$ & $34.8[72]$ & 0.4 \\
\hline Malaria diagnosis confirmed by medical team (\%) & 61.9 & 62.4 & 0.9 \\
\hline Fulfil WHO criteria (\%) & 17.8 & 17.8 & 0.9 \\
\hline
\end{tabular}

ACT: artemisinin combination therapy; AM: Argemone mexicana decoction.

a AM/ACT assignment ratio 2:1.

${ }^{\mathrm{b}}$ Axillary temperature in ${ }^{\circ} \mathrm{C}$.

${ }^{\mathrm{c}}$ Number of patients tested in square brackets.

and one had a P. ovale/P. vivax infection. The main medical diagnosis (taking account of each patient's history, physical examination and parasitaemia) was malaria in $62 \%$ of cases. The most common other diagnosis was a viral infection. In other words, $26 \%$ of patients were considered by the research team to have a viral or other non-malarial illness unlikely to be related to the low level of malaria parasites in their blood. Only $17.8 \%$ of patients in each group fulfilled all the WHO diagnostic criteria recommended for research on uncomplicated malaria ${ }^{21}$ (temperature $\geq 37.5^{\circ} \mathrm{C}$, parasitaemia $>2000 / \mathrm{mcl}$, and no concomitant infection that could be the cause of the fever).

Among patients coming from Missidougou village, 55\% consulted within $24 \mathrm{~h}$ of onset of symptoms, compared with $22 \%$ of patients coming from other villages. Some haematocrit values are missing because some of the capillary tubes burst open in the centrifuge.

\subsection{Dosage and compliance}

Although not every administration of the medicine was observed, patients were asked about their compliance with the prescribed treatment. In the AM group and the ACT group 75.1 and $98.0 \%$, respectively $(P<0.001)$, said they had taken the treatment at the dose prescribed. In the ACT group, $2 \%$ had taken a double dose on the first day of treatment. In the AM group, 5.5\% said that they took less than the dose prescribed, or had missed one or more doses; $18.3 \%$ said they had taken more than the dose prescribed. In terms of treatment duration, 55.3\% took the decoction for exactly 7 days; $7.1 \%$ took it for $<7$ days and $37.6 \%$ took it for $>7$ days ( $9.2 \%$ for $10-12$ days, $28.0 \%$ for $13-14$ days). Of those who prolonged their treatment with the decoction, $49 \%$ had been advised to do so. Prolongation of AM treatment during a second week was associated with second-line antimalarial treatment in the second fortnight (7/70 second-line among prolonged treatment, versus $3 / 117$ among nonprolonged; $P=0.05$ ). However if prolongation had not been possible, most of these patients would have needed a second-line treatment because of mild persistent symptoms.

Almost all patients used the preparation in both proposed routes of administration: drinking and washing (97.5\%; only one child and four adults did not wash). Use of adjuvant treatments was reported by $55.3 \%$ of patients in the AM group and $43.6 \%$ in the ACT group $(P=0.055)$ : principally paracetamol (AM $80.0 \%$, ACT $79.5 \%$, $P=0.95$ ), anti-emetics (AM $1.8 \%$, ACT $4.5 \%, P=0.32$ ), ginger (AM 10.9\%, ACT $11.4 \%, P=0.94$ ), antitussive preparation of Guiera senegalensis (AM 15.5\%, ACT 20.5\%, $P=0.1$ ) or others (AM $0 \%$, ACT $2 \%, P=0.14$ ).

\subsection{Clinical efficacy}

The proportion of patients not needing treatment with the second-line antimalarial remained under the set non-inferiority range, i.e. under the pre-defined minimally important difference (Table 2). Severe malaria developed in $1.9 \%$ of patients aged $\leq 5$ years in each group by day 28 (there were no cases in those aged $>5$ years). In both patients of the AM group, this consisted of anaemia and rapid breathing, and one had persistent vomiting. The one patient of the ACT group was repeatedly vomiting, unable to swallow the artesunateamodiaquine, and had worsening anaemia. The latter was the only patient requiring admission to hospital for parenteral antimalarials and a transfusion. Among all severe malaria cases (see Table 2), none had coma or convulsions, a definition used in other similar studies. ${ }^{22}$ No patients died in either group during the 28 days of follow-up.

It was already known that AM does not produce total parasite clearance in the majority of patients. ${ }^{16}$ The proportion of patients with parasitaemia at day 28 was $63-76 \%$ in the AM group and $21-49 \%$ in the ACT group (lower and upper estimates, $P<0.001$ with both estimates). Gametocytes were present at day 28 in $13 \%$ of the AM group compared with $3 \%$ of the ACT group $(P=0.005)$. However, 
Table 2

Outcomes of treatment of uncomplicated malaria by a village health worker with Argemone mexicana decoction (AM) or artemisinin combination therapy (ACT) as first-line antimalarial

\begin{tabular}{lll}
\hline & AM group [\% (95\% CI)] & ACT group [\% (95\% CI)] \\
\hline No need for second-line treatment & $89.3(84.1-93.2)$ & $95(88.8-98.3)$ \\
$\mathrm{T}<37.5^{\mathrm{a}}$ at day 14 & $93.9(89.3-96.7)$ & $97.0(91.6-99.4)$ \\
$\mathrm{T}<37.5^{\mathrm{a}}$ at day 28 & $0(0-1.83)$ & $99.0(94.6-100.0)$ \\
Severe malaria $>5$ years & $1.9(0.2-6.7)$ & $0(0-3.62)$ \\
Severe malaria (0-5 years) & $0(0-1.83)$ & $1.9(0.05-10.3)$ \\
Severe malaria (all ages): coma/convulsions & $14.2(9.7-19.9)^{\mathrm{b}}$ & $0(0-3.62)$ \\
Adverse effects & $12.8(8.4-18.3)$ \\
New episode (day 15-28) (parasite-positive) & $18.8(11.7-27.8)^{\mathrm{b}}$ \\
\hline
\end{tabular}

a Temperature in ${ }^{\circ} \mathrm{C}$.

${ }^{\mathrm{b}}$ Most common adverse effects: cough and diarrhoea with AM; vomiting with ACT.

the number of new parasite-positive episodes did not differ significantly between the groups (Table 2 ).

Among the $17.8 \%$ of patients meeting WHO diagnostic criteria for research on uncomplicated malaria, there was a clear difference between the groups at day 14, with $100 \%$ of patients on artesunate-amodiaquine achieving an 'adequate clinical response', compared with $65.7 \%$ on AM $(P=0.019)$. This was expected from previous study results. ${ }^{16}$ However, by day $28,17 \%$ of these patients on artesunate-amodiaquine had experienced a new episode, and $6 \%$ had a late treatment failure, leaving only $78 \%$ of patients on ACT with an adequate clinical response at day 28 . Of these, only $43 \%$ had a definite parasite clearance maintained at day 28 (no parasites seen by either microscopist).

There was no clinically significant difference in mean haematocrit between groups at day 28 (AM group 34.3, ACT group 35.7, median 34 and 36, $P=0.43$ ). However, this overall similarity does mask a few striking changes in individual cases: in particular in a small number of children whose haematocrit dropped after day 0 and required the second-line treatment.

\subsection{Safety and tolerability}

Overall, a similar proportion of patients experienced an adverse event in both groups, with a trend to fewer adverse events with AM (14.2\%) than with ACT (18.8\%). The majority of adverse events were deemed to be possibly, probably or very probably linked to the treatment (altogether $71 \%$ in the AM group, $79 \%$ in the ACT group; $P=0.67$ ). Only one patient in each group had to stop the treatment because of an adverse event, which in both cases was persistent vomiting.

Cough was significantly more common in the AM group ( 5.6 vs. $1 \%$ in the ACT group; $P=0.02$ ) and was the commonest adverse event in this group, along with diarrhoea. Nausea was significantly more common in the ACT group ( 8.9 vs. $2 \%$ in the AM group, $P=0.02$ ). There was also a trend towards more vomiting (8.9\%) and dizziness (2\%) in the ACT group (AM group: 3 and $0 \%$, respectively).

The adverse events started earlier on ACT than on AM (mean day 2.4 vs. $5.2, P=0.017$ ). The majority of adverse events $(89 \%)$ were graded as mild, and $11 \%$ were graded as moderate in both groups. There were no severe adverse events in either group.
Although not planned in the study protocol, a small additional survey was conducted towards the end of the study with the six team members and 12 villagers on preferences for one treatment strategy or another. This was done because of the non-blinded nature of the study design. The team members showed a tendency towards more preferences for the AM strategy than the villagers, whether for themselves ( 66 vs. $58 \%$ ) or for their child ( 83 vs. $42 \%$ ). Commonest reasons given for preference towards modern treatment were 'cures faster' and 'less work', whereas for AM treatment it was 'slower but better cure' and 'less adverse effects'.

\section{Discussion}

\subsection{Statement of principal findings}

This study, conducted in a remote village in southeast Mali, found that, with a decoction of Argemone mexicana as first-line treatment, 89\% (95\% CI 84.1-93.2) of patients did not require second-line treatment during the 28-day follow-up, compared with 95\% (95\% CI 88.8-98.3) among patients treated with ACT. Deterioration to severe malaria was $1.9 \%$ in both groups in children aged $\leq 5$ years (with $0 \%$ coma/convulsions) and $0 \%>5$ years. Overall, there were no significant differences between the groups in most of the outcome measures, and both treatments were well tolerated.

\subsection{Strengths and weaknesses of the study}

This is the largest comparative study of a herbal antimalarial to date, and is the only one to include children aged $<5$ years, and to include incidence of severe malaria as an outcome measure. The group random assignment worked well, as evidenced by the very similar baseline characteristics shown in Table 1. Follow-up was excellent, with no losses, thanks to proactive case-finding of all patients who did not return.

It was not possible to blind patients or clinicians to the treatment being taken. The dose of AM given to patients could not be standardised, as it was prepared at home. This is a reflection of what would happen in real life if a strategy with this treatment were adopted as a public-health intervention. Dose variation is acceptable if the therapeutic range is wide enough, which applies to AM. This wide 
therapeutic range was suspected from the first ethnomedical study, by the fact that the traditional dose regimen with AM is 'to drink as much as possible' of the preparation. The regimen chosen for this study was that with the best observed risk/benefit profile in the dose-escalating study. ${ }^{16}$ In addition, almost all patients also washed with the preparation.

Besides dosage, another important aspect of the standardisation is the phytochemical profile of the plants. In this study (awaiting the discovery of the active compounds for more precise quality control), the quality of the plant material was ensured by the principle of the 'controlled origin': all the plants used came from the region around the village where the tested recipe came from, to maximise the probability of a similar content between batches. Voucher specimens have been deposited at the Department of Traditional Medicine herbarium in Mali. Today, the 'médicament traditionnel amélioré' (improved traditional drug) is prepared following the same principle, with its raw materials coming from the same region. Even with a drug given with milligram or microgram precision, once it has been digested, metabolised and transported to the therapeutic target, the plasma concentration will also vary greatly between individuals.

The presence of a medical team in the village may have introduced a bias by improving compliance in both groups, ensuring the continued availability of all the necessary medications, and ensuring prompt referral to hospital for the few cases in which this was needed.

The study team found that it was necessary to prolong the treatment with the decoction for a second week in some patients, who were improving but were still experiencing some fever at day 7 . This suggests that 7 days might be too short as a general rule. Although re-treatment with the second-line antimalarial was more common in those who prolonged their treatment, these were patients who still had minor symptoms at day 7 (so were not randomly selected).

In the small subgroup of patients who fulfilled all the WHO research criteria for uncomplicated malaria, ${ }^{21}$ the outcomes were significantly better in the group receiving artesunate-amodiaquine. However, it should be stressed that this subgroup only accounted for $17.8 \%$ of patients in each group and is only a small subset of patients treated for presumptive 'malaria' where diagnostic facilities are limited. Use of such inclusion criteria would have led to conclusions that were not representative of the real situation. It is important to point out that the WHO diagnostic criteria for research are designed to have a very high specificity for clinical malaria (i.e. children under 5 years only, with temperature $\geq 37.5^{\circ} \mathrm{C}$, parasitaemia $>2000$, and no concomitant illness). They have a low sensitivity (they exclude many patients who would be diagnosed with malaria in a clinical setting), and are not the same as the WHO diagnostic criteria for treatment, ${ }^{7}$ which state that any patient with malaria parasites and fever should be treated for malaria. The latter also state that if parasitological diagnosis is not possible, the patient must be treated on the basis of a clinical diagnosis. On this last criterion, all our patients fitted a clinical diagnosis of malaria, in a village setting where parasitological diagnosis would not normally be available. Even in settings where a parasitological diagnosis is available, $87-88 \%$ of our patients (with positive thick film) would have fitted the criteria for treatment.

The classical 28-day follow-up might be too short in this case, as the lack of parasite clearance may predispose to later events.

\subsection{Strengths and weaknesses in relation to other studies, discussing important differences in results}

This is, to our knowledge, the first large-scale randomised controlled trial of a herbal medicine for the home-based management of malaria. Previous trials of the home-based management of malaria have used prepackaged drugs: usually chloroquine. One such trial was conducted in nearby Burkina Faso, where the epidemiology of malaria is very similar. ${ }^{22}$ Children $(<6$ years of age) who had had an episode of fever in the previous 4 weeks had an overall risk of developing severe malaria of $8 \%$. Severe malaria was defined in this study as' 'fever followed by convulsions or loss of consciousness'. The risk was reduced to $5 \%$ in those receiving pre-packaged chloroquine, compared to $11 \%$ in those not receiving this intervention. By contrast, in our study, the incidence of severe malaria by this definition was $0 \%$ in both groups. No patient developed convulsions or loss of consciousness in the 28 days of follow-up.

Although our results are not directly comparable with the study by Sirima et al.,22 because their study was retrospective and used a different treatment, both treatments tested in the present study were correlated with a lower incidence of severe malaria. The high prevalence of parasitaemia at day 28 in the ACT group could be a consequence of significant levels of amodiaquine resistance in this area.

\subsection{Meaning of the study: possible explanations and implications for clinicians and policymakers}

This study suggests that AM as first-line treatment may be capable of preventing severe malaria in a hightransmission malarious area. AM could be considered in pilot malaria programmes for semi-immune populations, in order to increase the rate of timely treatment and decrease the need for ACTs (with a consequent reduction in the risk of resistance). Delay in starting treatment is one of the main obstacles to achieving the Abuja target of $60 \%$ of patients being treated within $24 \mathrm{~h}$ of onset of symptoms. Typically under $50 \%$ of patients receive treatment within this time. ${ }^{23}$ In this study we found that $55 \%$ of patients sought treatment within $24 \mathrm{~h}$ in the village of the trial, compared with only $22 \%$ of patients who travelled from other villages. This emphasises the importance of homebased (or at least village-based) management if the target is to be met. From this study we can estimate that, in similar conditions, $89 \%$ (95\% CI 84.1-93.2) of patients could be treated with AM without needing a second-line treatment.

\subsection{Unanswered questions and future research}

In view of the fact that complete parasite clearance was not achieved in most patients treated with AM, the 
question remains whether the persistent parasitaemia at day 28 leads to an increase in new episodes of malaria, or in anaemia in subsequent weeks or months. It was decided that patients would therefore be followed up to 84 days, and the results of this follow-up will be the subject of a subsequent paper. It is also important to assess the cost-effectiveness of both treatment strategies, taking account of the treatment of subsequent episodes and alternative treatments where necessary.

For AM, 7 days of treatment might be too short as a general rule. In future studies a longer basic course of AM should be chosen: 10 or even 14 days. Most synthetic antimalarials (including amodiaquine, the partner drug in the ACT used in this study, and chloroquine) have a long halflife, staying in the bloodstream for at least 14 days.

Further research is ongoing to identify the active principle(s) in AM, in order to aid standardisation and quality control, which is especially important if this preparation is to be used in other areas.

\subsection{Conclusions}

In view of the low rate of observed severe malaria with AM treatment and its good safety profile, AM could now be tested in a pilot public-health programme as a first-line complement to standard modern drugs for semi-immune patients in high-transmission areas, in order to reduce the drug pressure for development of resistance. AM may also constitute a first-aid treatment when access to other antimalarials is delayed.

Authors' contributions: MLW, BG, JF and DD designed the study protocol; MLW and OS carried out the clinical assessment, laboratory analyses and follow-up; BG, MLW, CD, JF, FD, OS, SG and DD all worked on analysis and interpretation of the data; BG and MLW drafted the manuscript. All authors read and approved the final manuscript. BG and MLW are guarantors of the paper.

Acknowledgements: We wish to thank the people and elders of Missidougou, in particular chief Tiemoko Bengaly and his son Madou Bengaly, without whose open and enthusiastic collaboration this study would not have been possible. We are also very grateful to our fieldwork staff, Mr Diafara Berthé, Mr Yussuf Berthé and Dr Modibo Doumbia, for their hard work in the field. We would like to thank the staff and doctoral students of the Département de Médecine Traditionnelle, the Malian health authorities, the National Malaria Control Programme and the PSDS office in Sikasso, for their invaluable advice in the planning and analysis of this study, as well as André Rougemont, Bernard Burnand, Blaise Genton, Jean-Pierre Gervasoni, Steven Wayling and Franco Pagnoni in Switzerland.

Funding: The Swiss Agency for Development and Cooperation funded this study.

Conflicts of interest: None declared.

Ethical approval: Ethical clearance for this study was given by the Ethics Committee of the Institut National de
Recherche en Sante Publique (INRSP) of Mali on 6 June 2006. Note: when the study was being planned, there was no registration number in the ethical clearance system in Bamako.

\section{References}

1. Enosse $\mathrm{S}$, Magnussen $\mathrm{P}$, Abacassamo $\mathrm{F}$, Gomez-Olive $\mathrm{X}$, Ronn AM, Thompson R, et al. Rapid increase of Plasmodium falciparum dhfr/dhps resistant haplotypes, after the adoption of sulphadoxinepyrimethamine as first line treatment in 2002, in southern Mozambique. Malaria J 2008;7:115.

2. Mandi G, Mockenhaupt FP, Coulibaly B, Meissner P, Muller O. Efficacy of amodiaquine in the treatment of uncomplicated falciparum malaria in young children of rural north-western Burkina Faso. Malaria J 2008;7:58.

3. Martensson A, Stromberg J, Sisowath C, Msellem MI, Gil JP, Montgomery SM, et al. Efficacy of artesunate plus amodiaquine versus that of artemether-lumefantrine for the treatment of uncomplicated childhood Plasmodium falciparum malaria in Zanzibar, Tanzania [comment]. Clin Infect Dis 2005;41: 1079-86.

4. Breman JG. The ears of the hippopotamus: manifestations, determinants, and estimates of the malaria burden. Am J Trop Med Hyg 2001;64(1-2 Suppl):1-11.

5. WHO. The world malaria report. Geneva: World Health Organization 2005, WHO/HTM/MAL/2005.1102.

6. Gyapong M, Garshong B. Lessons learned in home management of malaria: implementation research in four African countries. Geneva: WHO/TDR Special Programme for Research and Training in Tropical Diseases; 2007.

7. WHO. Guidelines for the treatment of malaria. Geneva: World Health Organization; 2006, WHO/HTM/MAL/2006.1108.

8. Willcox ML, Sanogo F, Graz B, Forster M, Dackuo F, Sidibe O, et al. Rapid diagnostic tests for the home-based management of malaria in a high transmission area. Ann Trop Med Parasitol 2009;103: $3-16$.

9. Willcox ML, Bodeker G. Frequency of use of traditional herbal medicines for the treatment and prevention of malaria. In: Willcox M, Bodeker G, Rasoanaivo P, editors. Traditional medicine, medicinal plants and malaria. Boca Raton: CRC Press; 2004.

10. Graz B, Diallo D, Falquet J, Willcox M, Giani S. Screening of traditional herbal medicine: first, do a retrospective study, with correlation between diverse treatments used and reported patient outcome. $J$ Ethnopharmacol 2005;101:338-9.

11. Diallo D, Graz B, Falquet J, Traore AK, Giani S, Mounkoro PP, et al. Malaria treatment in remote areas of Mali: use of modern and traditional medicines, patient outcome. Trans $\mathrm{R}$ Soc Trop Med Hyg 2006;100:515-20.

12. Emmart EW. The Badianus Manuscript: an Aztec herbal of 1552. Baltimore, MD: Johns Hopkins Press; 1940.

13. Adjobimey T, Edayé I, Lagnika L, Gbenou J, Moudachirou M, Sanni A. Activités antiplasmodiales in vitro de quelques plantes antipaludiques de la pharmacopée béninoise. $C R$ Chimie 2004;7:1023-7.

14. Diallo D, Mounkoro PP, Sangaré D, Graz B, Falquet J, Giani S, et al. La prise en charge du paludisme par les thérapeutes traditionnels dans les aires de santé de Kendié (Bandiagara) et de Finkolo (Sikasso) au Mali. Mali Med 2007;12:1-8

15. Guindo M., Contribution à l'étude du traitement traditionnel du "suma" (paludisme) [thèse pour obtenir le grade de Docteur en Pharmacie]. Bamako, Mali: Ecole Nationale de Médecine et de Pharmacie du Mali; 1988.

16. Willcox ML, Graz B, Falquet J, Sidibe O, Forster M, Diallo D Argemone mexicana decoction for the treatment of uncomplicated falciparum malaria. Trans $R$ Soc Trop Med Hyg 2007;101 1190-8.

17. Dumville JC, Hahn S, Miles JN, Torgerson DJ. The use of unequal randomisation ratios in clinical trials: a review [comment]. Contemp Clin Trials 2006;27:1-12.

18. Pagnoni F, Convelbo N, Tiendrebeogo J, Cousens S, Esposito F. A community-based programme to provide prompt and adequate treatment of presumptive malaria in children. Trans $R$ Soc Trop Med Hyg 1997;91:512-7.

19. Hopkins H, Talisuna A, Whitty CJ, Staedke SG. Impact of home-based management of malaria on health outcomes in Africa: a systematic review of the evidence. Malaria J 2007;6:134. 
20. WHO. General guidelines for methodologies on research and evaluation of traditional medicine. Geneva: World Health Organization; 2000.

21. WHO. Assessment and monitoring of antimalarial drug efficacy for the treatment of uncomplicated falciparum malaria. Geneva: World Health Organization; 2003, WHO/HTM/RBM/ 2003.50 .
22. Sirima SB, Konate A, Tiono AB, Convelbo N, Cousens S, Pagnoni F. Early treatment of childhood fevers with pre-packaged antimalarial drugs in the home reduces severe malaria morbidity in Burkina Faso. Trop Med Int Health 2003;8:133-9.

23. Nsungwa-Sabiiti J, Tomson G, Pariyo G, Ogwal-Okeng J, Peterson S. Community effectiveness of malaria treatment in Uganda-a long way to Abuja targets. Ann Trop Paediatr 2005;25:91-100. 